\title{
Calcium alginate hydrogel beads with high stiffness and extended dissolution behaviour
}

\begin{abstract}
Alginate hydrogel bead has been widely explored as a vehicle for controlled delivery application due to its non-toxicity, renewability, and ease of formation. However, alginate hydrogel beads are known to have a low stiffness, i.e., Young modulus $<1 \mathrm{MPa}$, and a short dissolution time of between $1 \mathrm{~h}$ and $2 \mathrm{~h}$ in gastrointestinal fluid. This study aimed to fabricate calcium alginate hydrogel beads with desired properties like high stiffness and extended dissolution behaviour. A temperature-controlled extrusionï dripping method incorporating an immiscible interphase column was used to produce the ultra-high concentration (UHC) calcium hydrogel beads directly from unmodified alginate solution. The UHC beads have an extraordinary internal structure with thick calcium-alginate matrices and large pores in between the matrices. The Youngôs modulus value of UHC calcium alginate beads was 3.6 $\mathrm{MPa}$, which was approximately 8 times higher than the normal calcium alginate beads. The release profile for the model drug (i.e., methylene blue) encapsulated in UHC beads was found to be extended to $4 \mathrm{~h}$ at $80 \%$ of drug release (t80). The kinetics of drug release fitted well with the Korsmeyerï Peppas model ( $\mathrm{r} 2 \geqslant 0.99)$ and followed the non-Fickian mechanism. These findings show that the preparation of calcium alginate beads featuring high stiffness and extended dissolution profile can be achieved without any chemical modification or additives. The UHC calcium alginate bead holds excellent promise as an encapsulation carrier of drugs or food used in controlled delivery applications.
\end{abstract}

Keyword: Alginate; Hydrogel; Extrusion; Stiffness; Controlled-release; Kinetics 